\title{
An Open Source Toolkit to Parse and Analyze Online Crowdsourced Portals
}

\author{
Amit Arjun Verma ( $\boldsymbol{D}$ 2016csz0003@iitrpr.ac.in ) \\ Indian Institute of Technology Ropar https://orcid.org/0000-0002-2392-0198 \\ S.R.S lyengar \\ Indian Institute of Technology Ropar \\ Simran Setia \\ Indian Institute of Technology Ropar \\ Neeru Dubey \\ Indian Institute of Technology Ropar
}

Research

Keywords: Knowledge Building, Wikipedia, Stack Exchange, open-source, Python library

Posted Date: January 29th, 2021

DOI: https://doi.org/10.21203/rs.3.rs-154658/v1

License: (9) This work is licensed under a Creative Commons Attribution 4.0 International License.

Read Full License 


\section{Abstract}

With the success of crowdsourced portals, such as Wikipedia, Stack Overflow, Quora, and GitHub, a class of researchers is driven towards understanding the dynamics of knowledge building on these portals. Even though collaborative knowledge building portals are known to be better than expert-driven knowledge repositories, limited research has been performed to understand the knowledge building dynamics in the former. This is mainly due to two reasons; first, unavailability of the standard data representation format, second, lack of proper tools and libraries to analyze the knowledge building dynamics.

We describe Knowledge Data Analysis and Processing Platform (KDAP), a programming toolkit that is easy to use and provides high-level operations for analysis of knowledge data. We propose Knowledge Markup Language (Knol-ML), a generic representation format for the data of collaborative knowledge building portals. KDAP can process the massive data of crowdsourced portals like Wikipedia and Stack Overflow efficiently. As a part of this toolkit, a data-dump of various collaborative knowledge building portals is published in Knol-ML format. The combination of Knol-ML and the proposed open-source library will help the knowledge building community to perform benchmark analysis.

\section{Full Text}

Due to technical limitations, full-text HTML conversion of this manuscript could not be completed. However, the manuscript can be downloaded and accessed as a PDF.

\section{Figures}




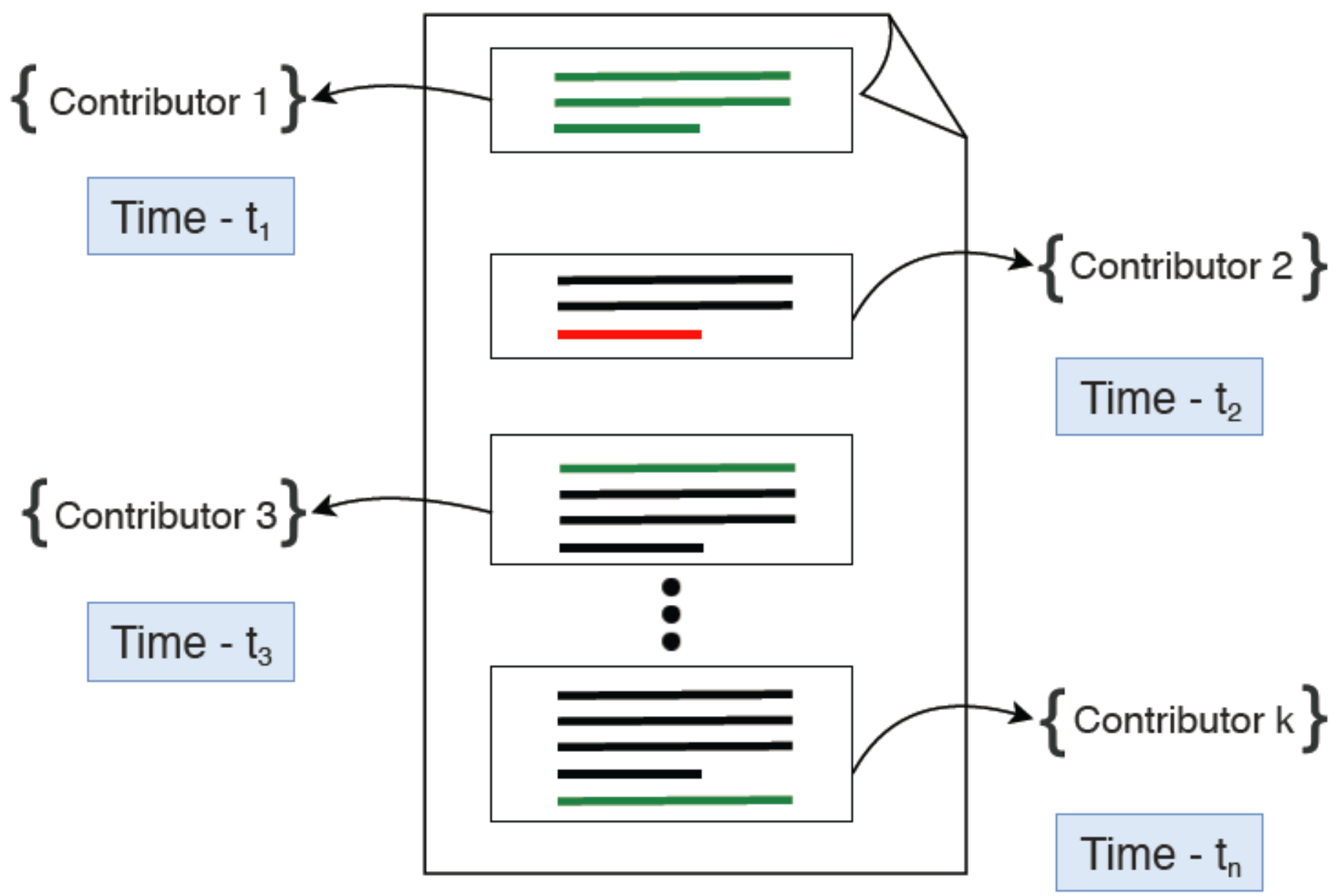

Figure 1

An illustration of sequential knowledge building in crowdsourced environ- ment. A contribution can be seen in the form of addition (green) or deletion (red) information. 
<KnowledgeData Type="text" Id="1">

<Title>This is an example for sequential knowledge data</Title>

<Instance Id="1" InstanceType="Revision">

<Timestamp><CreationDate>t $1</$ CreationDate $</$ Timestamp $>$

<Contributors>

<OwnerUserName>Editor 1</OwnerUserName>

<OwnerUserId>1</OwnerUserId>

$<$ /Contributors $>$

$<$ Body $><$ Text Type="text" $>$ Edits of Editor $1</$ Text $></$ Body $>$ $</$ Instance $>$

<Instance Id="2" InstanceType="Revision">

$<$ Timestamp $><$ CreationDate $>$ t $2</$ CreationDate $></$ Timestamp $>$

<Contributors>

<OwnerUserName>Editor 2</OwnerUserName>

<OwnerUserId >2</OwnerUserId>

$</$ Contributors $>$

$<$ Body $>\langle$ Text Type="text" $>$ Edits of Editor $2</$ Text $></$ Body $>$ $</$ Instance $>$

$</$ KnowledgeData $>$

Figure 2

Representation of sequential knowledge data in Knol-ML format. 
<Def attr.name="abc" attr.type="string" for="Instance" id="abc" />

<KnowledgeData Type="text" Id="1">

<Title>Title of the article</Title>

<Instance Id="1" InstanceType="Revision">

$\langle$ TimeStamp $><$ CreationDate $>$ t $1</$ CreationDate $>/$ TimeStamp $>$ Additional

<Contributors>

<OwnerUserName>Editor $1<$ /OwnerUserName>

<OwnerUserId >1</OwnerUserId>

$</$ Contributors $>$

<EditDetails>

<EditType>details of edit</EditType>

$</$ EditDetails $>$

$\langle$ Body $\rangle\langle$ Text Type="text" $>$ Edits of Editor $1</$ Text $\rangle\langle$ Body $\rangle$

Data

<Knowl Def="abc">sample value</Knowl>

$</$ Instance $>$

:

$</$ KnowledgeData $>$

Figure 3

Representation of extension mechanism in Knol-ML.

<KnowledgeData Type="text" Id="1">

<Title> Question Title </Title>

<Instance Id="1" InstanceType="Question"> <Timestamp>

<CreationDate> t1 </CreationDate>

$</$ Timestamp $>$

<Contributors>

<OwnerUserId> Editor 1 </OwnerUserId>

$<$ /Contributors $>$

$\langle$ Body $\rangle\langle$ Text Type="text" $\rangle$ Question $\langle/$ Text $\rangle\langle/$ Body $\rangle$ $<$ Credit $>\langle$ Score $>$ score value $</$ Score $>/$ Credit $>$ $</$ Instance $>$

$</$ KnowledgeData $>$

Figure 4 
A diagram of knowledge data structures. Instance ids are stored in a hash table, and each instance has one hash table and one vector associated with it representing the elements of the instance.

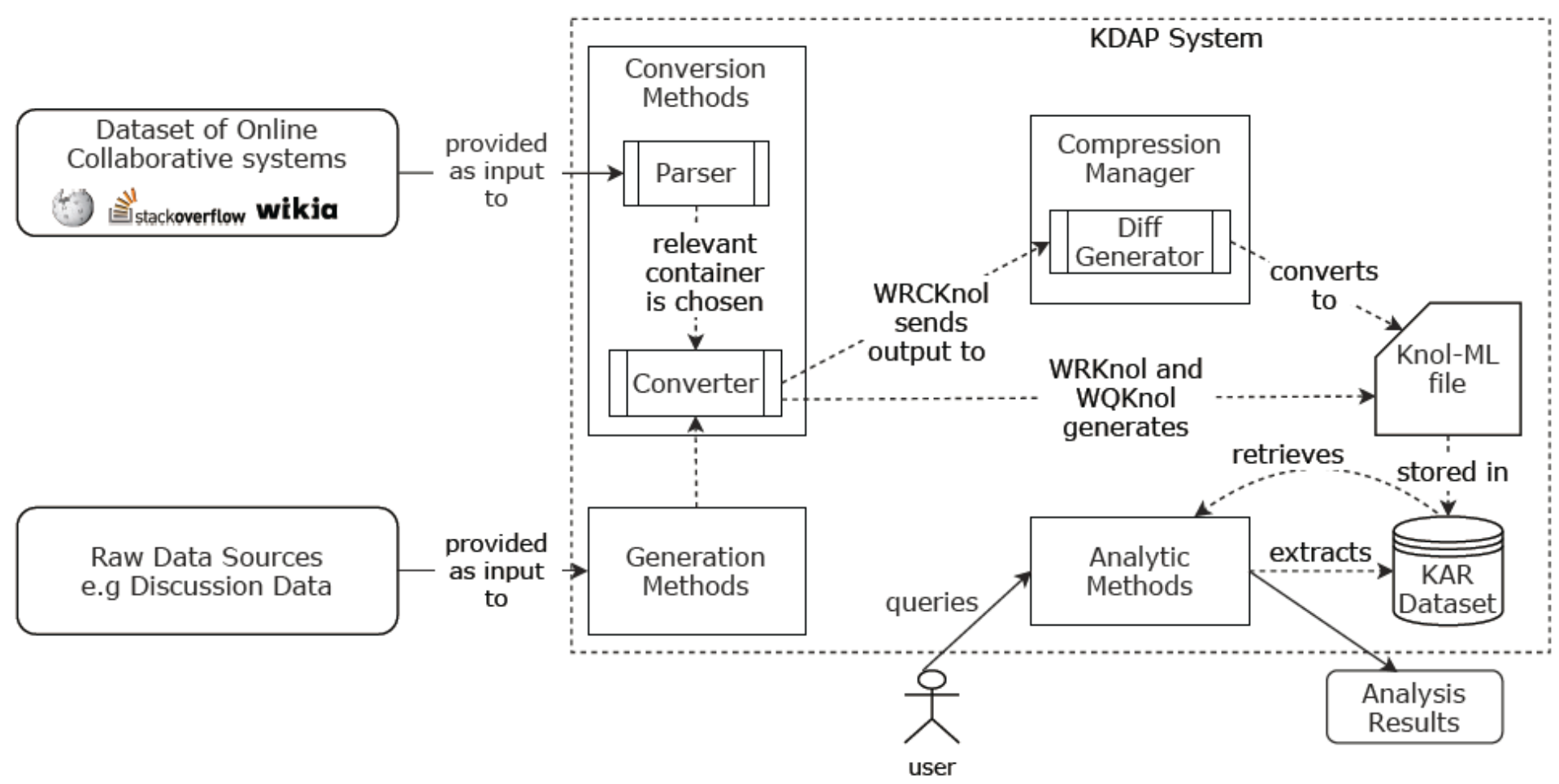

Figure 5

Overview of KDAP framework. 